\title{
SPARSE ENDPOINT ESTIMATES FOR BOCHNER-RIESZ MULTIPLIERS ON THE PLANE
}

\author{
ROBERT KESLER AND MICHAEL T. LACEY
}

\begin{abstract}
For $0<\lambda<\frac{1}{2}$, let $B_{\lambda}$ be the Bochner-Riesz multiplier of index $\lambda$ on the plane. Associated to this multiplier is the critical index $1<p_{\lambda}=\frac{4}{3+2 \lambda}<\frac{4}{3}$. We prove a sparse bound for $B_{\lambda}$ with indices $\left(p_{\lambda}, q\right)$, where $p_{\lambda}^{\prime}<q<4$. This is a further quantification of the endpoint weak $L^{p_{\lambda}}$ boundedness of $B_{\lambda}$, due to Seeger. Indeed, the sparse bound immediately implies new endpoint weighted weak type estimates for weights in $A_{1} \cap R H_{\rho}$, where $\rho>\frac{4}{4-3 p_{\lambda}}$.
\end{abstract}

\section{INTRODUCTION}

We establish a range of sparse bounds for the Bochner-Riesz multipliers in the plane, at the critical index of $p$. The Bochner-Riesz multipliers in the plane are defined through the Fourier transform by

$$
\mathcal{F}\left(B_{\lambda} f\right)(\xi)=\left(1-|\xi|^{2}\right)_{+}^{\lambda} \mathcal{F} f(\xi), \quad 0<\lambda<\frac{1}{2} .
$$

The case of $\lambda=\frac{1}{2}$ is the critical case in dimension $d=2$, where $B_{\frac{1}{2}}$ maps $L^{p}$ to itself for all $1<p<\infty$, and is of weak-type $(1,1)$. For $0<\lambda<\frac{1}{2}$, the critical index of $p_{\lambda}$ is given by

$$
\text { (1.1) } p_{\lambda}=\frac{4}{3+2 \lambda} \text {. }
$$

The Carleson-Sjölin theorem gives the $L^{p}$ boundedness of the $B_{\lambda}$.

Theorem A. [2] For $0<\lambda<\frac{1}{2}$, there holds

$$
\left\|B_{\lambda}\right\|_{p \rightarrow p}<\infty, \quad p_{\lambda}<p<p_{\lambda}^{\prime}=\frac{p_{\lambda}}{p_{\lambda}-1} .
$$

We recall the notion of a sparse bound, which are a particular quantification of the (weak) $L^{p}$-bounds for an operator. They in particular immediately imply weighted and vector-valued inequalities. Given a sublinear operator $T$, and $1 \leq r, s<\infty$, we set $\|T:(r, s)\|$ to be the infimum over constants $C$ so that for all measurable, bounded, and compactly supported functions $f, g$,

$$
|\langle T f, g\rangle| \leq C \sup \sum_{Q \in \mathcal{S}}\langle f\rangle_{Q, r}\langle g\rangle_{Q, s}|Q| .
$$

Research supported in part by grant from the US National Science Foundation, DMS-1600693 and the Australian Research Council ARC DP160100153. 


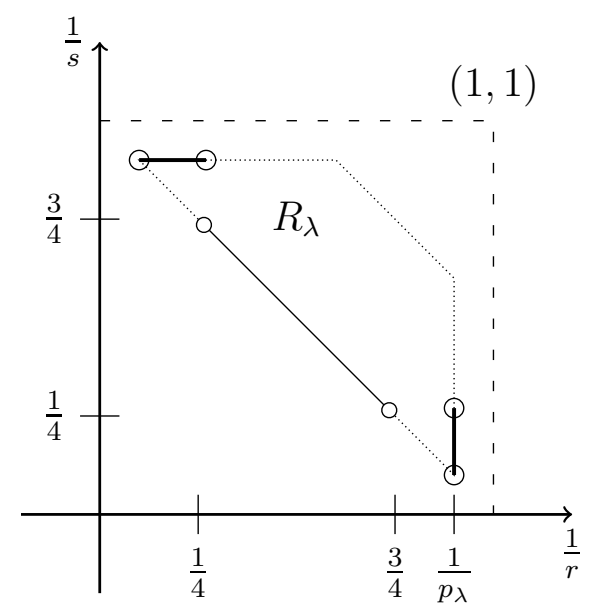

Figure 1. The solid diagonal line indicates the Carleson-Sjölin bounds. The dotted rhombus $R_{\lambda}$ is the open region for which a sparse bound holds for $B_{\lambda}$, see [8]. The solid vertical line above $\frac{1}{p_{\lambda}}$ indicates the range of sparse bounds proved in this paper from which the solid horizontal line is obtained as an immediate consequence. We leave it as a question if $B_{\lambda}$ satisfes a sparse bound for $\left(p_{\lambda}, s\right)$, where $\frac{1}{4} \leq \frac{1}{s}<$ $\frac{1+6 \lambda}{4}$.

We use throughout the notation $\langle f\rangle_{Q, r}=\left[|Q|^{-1} \int_{Q}|f|^{r} d x\right]^{\frac{1}{r}}$. The supremum above is formed over all sparse collections $\mathcal{S}$ of cubes. A collection $\mathcal{S}$ is sparse if for each $Q \in \mathcal{S}$ there is a set $E_{Q} \subset S$ so that (a) $\left|E_{Q}\right| \geq \frac{1}{10}|Q|$, and (b) the sets $\left\{E_{Q}: Q \in \mathcal{S}\right\}$ are pairwise disjoint. It is essential that the sparse form be allowed to depend upon $f$ and $g$. But the point is that the sparse form itself varies over a class of operators with very nice properties.

The sparse variant of the Carleson-Sjölin bounds has been studied in $[1,8]$.

Theorem B. [8] For $0<\lambda<\frac{1}{2}$, let $R_{\lambda}$ be the open rhombus with vertices

$$
\left(\frac{1}{p_{\lambda}}, \frac{1}{p_{\lambda}^{\prime}}\right),\left(\frac{1+6 \lambda}{4}, \frac{1}{p_{\lambda}^{\prime}}\right),\left(\frac{1}{p_{\lambda}}, \frac{1+6 \lambda}{4}\right),\left(\frac{1}{p_{\lambda}^{\prime}}, \frac{1}{p_{\lambda}}\right) .
$$

There holds

$$
\left\|B_{\lambda}:(r, s)\right\|<\infty, \quad\left(\frac{1}{r}, \frac{1}{s}\right) \in R_{\lambda}
$$

Moreover, the inequality above fails for $\frac{1}{r}+\frac{1}{s} \geq 1$, with $\left(\frac{1}{r}, \frac{1}{s}\right)$ not in the closure of $R_{\lambda}$.

The rhombus $R_{\lambda}$ is pictured in Figure 1. At the endpoint in the Carleson-Sjölin theorem, a weak-type estimate holds, as was proved by Seeger [9], extending the work of Christ [4]. 
Theorem C. For $0<\lambda<\frac{1}{2}$, there holds

$$
\left\|B_{\lambda}\right\|_{p_{\lambda} \rightarrow\left(p_{\lambda}, \infty\right)}<\infty .
$$

Our main result is a sparse quantification of this last result.

Theorem 1.2. Let $0<\lambda<\frac{1}{2}$, and $p_{\lambda}$ as in (1.1), these sparse bounds hold.

$$
\left\|B_{\lambda}:\left(p_{\lambda}, q\right)\right\|<\infty, \quad 4<q<p_{\lambda}^{\prime}=\frac{p_{\lambda}}{p_{\lambda}-1} .
$$

An immediate consequence of Theorem 1.2 is that $\left\|B_{\lambda}:\left(q, p_{\lambda}\right)\right\|<\infty$ for the same range of $q$ and $\lambda$. While the sparse bounds in Theorem B are sharp, up to the boundary, we could not determine sharpness above. Whether $\left\|B_{\lambda}:\left(p_{\lambda}, q\right)\right\|<\infty$ holds for $0<\lambda<\frac{1}{2}$ and $\frac{4}{1+6 \lambda}<q \leq 4$ remains an open question.

Our main result contains Seeger's inequality, as well as some weighted inequalities. While this paper is not the first to show weighted endpoint estimates for the BochnerRiesz multipliers, the literature on this subject is limited. While Vargas proves that Bochner-Riesz multipliers at the critical index $\frac{n-1}{2}$ are of weak type $(1,1)$ with respect to any weight in the $A_{1}$-Muckenhoupt class [11], weighted inequalities for BochnerRiesz multipliers below the critical index are new to our knowledge.

Corollary 1.3. Let $0<\lambda<\frac{1}{2}$ and $\rho>\frac{4}{4-3 p_{\lambda}}$. Then, for every $w \in A_{1} \cap R H_{\rho}$ we have the inequality

$$
\left\|B_{\lambda}\right\|_{L^{p_{\lambda}}(w) \mapsto L^{p_{\lambda}, \infty}(w)}<\infty .
$$

The implied constant depends only on the $A_{1}$ and reverse Hölder characteristic of $w$.

The notation above is standard. By [6, Thm 1.3], a quantitative version of Corollary 1.3 is that for $0<\lambda<\frac{1}{2}, \rho>\frac{4}{4-3 p_{\lambda}}$, and $w \in A_{1} \cap R H_{\rho}$,

$$
\left\|B_{\lambda}\right\|_{L^{p_{\lambda}}(w) \mapsto L^{p_{\lambda}, \infty}(w)} \leq c(n, \rho)\left[w^{\rho}\right]_{A_{\infty}}^{1+\frac{1}{p_{\lambda}}}\left([w]_{A_{1}}[w]_{R H_{\rho}}\right)^{\frac{1}{p_{\lambda}}} .
$$

The study of sparse bounds for the Bochner-Riesz multipliers was initiated by Benea, Bernicot and Luque [1]; their analysis was recently simplified and extended by one of us, Mena, and Reguera [8]. The reader should consult that paper for more background and references to the sparse literature.

\section{LEMMAS}

To align our discussion with the notation of [9], we expand Bochner-Riesz operator in the following way. For integers $j \geq 0$, let $T_{j}$ be a smooth radial Fourier projection adapted to $\left\{|\vec{\xi}|<1: \operatorname{dist}\left(\vec{\xi}, \mathbb{S}^{1}\right) \simeq 2^{-j}\right\}$. Specifically, pick $\phi \in C_{0}^{\infty}([-2,2])$ such that $\phi \equiv 1$ on $[0,1]$ and set $\psi(\xi)=\phi(\xi)-\phi(2 \xi)$ along with $\psi_{j}(\xi)=\psi\left(2^{j} \xi\right)$ for every integer $j \geq 0$. Then $\sum_{j \geq 0} \psi_{j}(\xi) \equiv 1$ on $[0,1]$, and consequently $\left(1-|\xi|^{2}\right)_{+}^{\lambda}=$ 
$\sum_{j \geq 0}\left(1-|\xi|^{2}\right)_{+}^{\lambda} \psi_{j}(1-|\xi|)$. Defining $\widehat{T_{j}^{\lambda} f}(\xi)=2^{\lambda j}\left(1-|\xi|^{2}\right)_{+}^{\lambda} \psi_{j}(1-|\xi|) \hat{f}(\xi)$, we will have for every $\lambda \in\left(0, \frac{1}{2}\right)$

$$
B_{\lambda}=T_{0}^{\lambda}+\sum_{j \geq 1} 2^{-\lambda j} T_{j}^{\lambda} .
$$

As $\left|T_{0}^{\lambda} f(x)\right| \lesssim M_{H L} f(x)$ for all locally integrable functions $f$ and $\left\|M_{H L}:(1,1)\right\|<\infty$, it suffices to prove Theorem 1.2 with $B_{\lambda}$ replaced by $\sum_{j \geq 1} 2^{-\lambda j} T_{j}^{\lambda}$. We shall need the following inequality concerning the kernel of $T_{j}^{\lambda}$.

Lemma 2.1. Set $T_{j}^{\lambda} f(x)=\tau_{j}^{\lambda} * f(x)$. For all integers $j, N \geq 1$ and $\lambda \in\left(0, \frac{1}{2}\right)$,

$$
\left|\tau_{j}^{\lambda}(x)\right| \lesssim_{N}\left[2^{-j}|x|\right]^{-2 N} 2^{-\frac{3 j}{2}}, \quad|x| \geq 2^{j} .
$$

While the implied constant depends upon $N$, it is uniform in $j \geq 0$ and $\lambda \in[0,1 / 2]$.

We could not find a source for this estimate, so include a proof here.

Proof. Fix $\lambda \in\left(0, \frac{1}{2}\right)$ and let $\tau_{j}^{\lambda}=\tau_{j}$. Then the Fourier transform of $\tau_{j}, \widehat{\tau_{j}}$, is a radial function, so that it follows from [10, Chap VII 5.12] that for the radial variable $u>0$, we have

$$
\tau_{j}(u)=2 \pi \int_{0}^{\infty} \widehat{\tau_{j}}(s) J_{0}(2 \pi u s) s d s
$$

where $J_{0}$ is the 0 th order Bessel function. For an integer $N$, apply integration by parts $2 N$ times, integrating the Bessel function each time. A complication arises here due to the presence of the Bessel functions, which is addressed in Lemma 2.3.

We have, using a change of variables and (2.4)

$$
\left|\int_{0}^{\infty} \widehat{\tau}_{j}(s) J_{0}(2 \pi u s) s d s\right|=(2 \pi u)^{-2 N}\left|\int_{0}^{\infty}\left[M^{-1} D M D\right]^{N} \widehat{\tau}_{j}(s) \cdot J_{0}(2 \pi u s) s d s\right| .
$$

Examine the integral above. The function $\left[M^{-1} D M D\right]^{N} \widehat{\tau}_{j}(s)$ is supported in an interval close to $s=1$ of width $2^{-j}$. Each derivative applied to $\widehat{\tau_{j}}(s)$ gains a factor of $2^{j}$, so that the function is bounded bounded by $C_{N} 2^{2 j N}$. And, the asymptotics for the Bessel function are

$$
\left|J_{0}(2 \pi u)\right| \lesssim u^{-\frac{1}{2}} \lesssim C_{N} 2^{-\frac{j}{2}}
$$

Therefore we have the estimate below, which completes the Lemma.

$$
\left|\tau_{j}(u)\right| \leq C_{N}\left[u^{-1} 2^{j}\right]^{2 N} \cdot 2^{-\frac{3 j}{2}} .
$$

Lemma 2.3. Let $\chi$ be a smooth compactly supported function on $(0, \infty)$. We have

$$
\int_{0}^{\infty} \chi(s) J_{0}(s) s d s=-\int\left[M^{-1} D M D \chi(s)\right] J_{0}(s) s d s,
$$

where $D=\frac{d}{d x}$ and $M \phi(s)=s \phi(s)$ is multiplication by the independent variable. 
Proof. There are two classical identities concerning the Bessel functions, that we need. Namely,

$$
\begin{aligned}
D^{-1} s J_{0}(s) & =s J_{1}(s), \\
D^{-1} J_{1} & =-J_{0} .
\end{aligned}
$$

Using integration by parts, (2.5) and (2.6), in that order we have

$$
\begin{aligned}
\int_{0}^{\infty} \chi(s) \cdot s J_{0}(s) d s & =-\int D \chi(s) \cdot s J_{1}(s) d s \\
& =-\int M D \chi(s) \cdot J_{1}(s) d s \\
& =-\int D M D \chi(s) \cdot J_{0}(s) d s \\
& =-\int M^{-1} D M D \chi(s) \cdot s J_{0}(s) d s
\end{aligned}
$$

This completes the proof.

The $L^{p}$ mapping properties of $T_{j}$ are well known, namely

$$
\left\|T_{j}\right\|_{p \rightarrow p} \lesssim \begin{cases}2^{\frac{j}{2}} & p=1 \\ 2^{\lambda_{p} j} & 1<p<\frac{4}{3} . \\ j^{\frac{1}{4}} & p=\frac{4}{3}\end{cases}
$$

The first is well known, the second is a consequence of Hörmander's version of the Carleson-Sjölin bounds from [7], and the third is due to Córdoba [5]. These estimates are not sufficient for the endpoint case, however. Our proof implicitly relies upon the Theorem below, a key inequality of Seeger [9] . We will appeal to certain consequences of it.

Theorem 2.8. [9, Thm 2.1] Suppose that $1 \leq p<\frac{4}{3}$ and $\lambda_{p}=2\left(\frac{1}{p}-\frac{1}{2}\right)-\frac{1}{2}$. Then there is the inequality

$$
\left\|\sum_{j=1}^{\infty} T_{j}^{\lambda_{p}} f_{j}\right\|_{p} \lesssim\left[\sum_{j=1}^{\infty} 2^{p \lambda_{p} j}\left\|f_{j}\right\|_{p}^{p}\right]^{\frac{1}{p}} .
$$

Technically, Theorem 2.1 in [9] yields (2.9) for the family $\left\{S_{j}\right\}_{j \geq 1}$ where $\widehat{S_{j} f}(\xi)=$ $\eta(\xi)\left(1-|\xi|^{2}\right)_{+}^{\lambda_{p}} \psi_{j}(1-|\xi|) \hat{f}(\xi)$ where $\eta \in C_{0}^{\infty}\left(\mathbb{R}^{2}\right)$ has $\operatorname{supp}(\eta) \subset\left\{\xi \in \mathbb{R}^{2}:\left|\xi_{1} / \xi_{2}\right| \leq\right.$ $\left.10^{-1}, \xi_{2}>0\right\}$ and $\psi_{j}$ is the same function as before. However, rotating the Fourier transform of $\left\{f_{j}\right\}$ yields $(2.9)$ for $\eta$ precomposed with any rotation. It is then straightforward to rewrite for any $j \geq 1$

$$
\psi_{j}(1-|\xi|)=\sum_{k=1}^{K} \eta_{k}(\xi) \psi_{j}(1-|\xi|)
$$


where $K=O(1)$ and each $\eta_{k}=\tilde{\eta}_{k} \circ R_{k}$ where $R_{k}$ is a rotation and $\tilde{\eta}_{k} \in C_{0}^{\infty}\left(\mathbb{R}^{2}\right)$ with $\operatorname{supp}\left(\tilde{\eta}_{k}\right) \subset\left\{\xi \in \mathbb{R}^{2}:\left|\xi_{1} / \xi_{2}\right| \leq 10^{-1}, \xi_{2}>0\right\}$. This naturally leads to a decomposition $T_{j}^{\lambda_{p}}=\sum_{k=1}^{K} T_{j, k}^{\lambda_{p}}$. By the triangle inequality and the validity of (2.9) on the $\left\{T_{j, k}^{\lambda_{p}}\right\}_{j \geq 1}$ for each $1 \leq k \leq K$ yields (2.9) for $\left\{T_{j}^{\lambda_{p}}\right\}_{j \geq 1}$ as claimed. The restriction $1 \leq p<\frac{4}{3}$ above, will by duality, lead to the restriction that $s>4$ in our main theorem.

\section{PROOF}

The method of proof begins with the analysis of Seeger [9], and finishes with some additional arguments. The analysis depends upon a crucial endpoint estimate Theorem 2.8 above, and a fine Calderón-Zygmund analysis, in the style of Christ $[3,4]$.

To state the main recursive estimate needed in the proof, further set a truncated Bochner-Riesz operator adapted to any dyadic square $Q$ to be

$$
T^{\lambda_{p}, Q} f=\sum_{j: 2^{j}<\ell Q} 2^{-\lambda_{p} j} T_{j}^{\lambda_{p}} f, \quad \lambda_{p}=2\left(\frac{1}{p}-\frac{1}{2}\right)-\frac{1}{2} .
$$

Lemma 3.1. Let $1<p<\frac{4}{3}, 4<q<p^{\prime}$. For any square $E$ and pair of measurable and bounded functions $f: E \rightarrow \mathbb{C}$ and $h: 3 E \rightarrow \mathbb{C}$ there is a collection $\mathcal{B}$ of disjoint subsquares of $E$ with the properties $\left|\bigcup_{Q \in \mathcal{B}} Q\right| \leq \frac{|E|}{100}$ and

$$
\left|\left\langle T^{\lambda_{p}, E} f, h\right\rangle\right| \lesssim\langle f\rangle_{E, p}\langle h\rangle_{3 E, q}|E|+\sum_{Q \in \mathcal{B}}\left|\left\langle T^{\lambda_{p}, Q}\left(f 1_{Q}\right), \mathbf{1}_{3 Q} h\right\rangle\right| .
$$

Below, we will cite two inequalities for the Bochner-Riesz operators from the work of Seeger [9]. But, our application of the estimates differs from their form in [9] in that we will have a truncation $T^{E}$ in place of the Bochner-Riesz operator. These new inequalities hold, with the same proof as in [9]. This can be seen from the fact that the crucial vector-valued inequality [9, Thm 2.1] trivially extends to the truncated case. And, all of the endpoint interpolation estimates in [9, (3.5-3.14)] extend uniformly.

Proof. Fixing $p \in\left(1, \frac{4}{3}\right)$, let $T_{j}=T_{j}^{\lambda_{p}}$ for all integers $j \geq 1$ and let $T^{Q}=T^{\lambda_{p}, Q}$ for all dyadic squares $Q$. If $E$ has area one, the Lemma holds with $\mathcal{B}$ being empty. Assume therefore that $E$ has area larger than one. The following argument is not symmetric, in that we perform an $L^{p}$ Calderón-Zygmund decomposition on $f$ at level $\langle f\rangle_{E, p}$, while the function $h$ remains untouched. This yields $f=g+b$ where $\|g\|_{L^{\infty}} \lesssim\langle f\rangle_{E, p}$ and $\|g\|_{p} \lesssim\|f\|_{p}$. The function $b$ satisfies $b=\sum_{Q \in \mathcal{B}} b_{Q}$, where $\mathcal{B}$ are disjoint dyadic subsquares of $E$, with

$$
\left|\bigcup_{Q \in \mathcal{B}} Q\right| \leq \frac{1}{100}|E|
$$

The functions $b_{Q}=\left(f-\langle f\rangle_{Q}\right) \mathbf{1}_{Q}$ are supported on $Q$, and satisfy $\left\|b_{Q}\right\|_{p}^{p} \lesssim\langle f\rangle_{E, p}^{p}|Q|$. Here, $\langle f\rangle_{Q}=|Q|^{-1} \int_{Q} f d x$. 
The function $b$ is then further decomposed into the sum $\sum_{t=0}^{\infty} \beta_{t}$, where the index $t$ indexes the side lengths of the squares $Q \in \mathcal{B}$. Namely,

$$
\beta_{0}:=\sum_{Q \in \mathcal{B}: \ell Q \leq 0} b_{Q}, \quad \text { and } \beta_{t}:=\sum_{Q \in \mathcal{B}: \ell Q=2^{t}} b_{Q}, 0<t<\log _{2} \ell E
$$

where $\ell Q$ and $\ell E$ are the sidelengths of $Q$ and $E$ respectively. We then have the equality below for the truncated Bochner-Riesz operator applied to $b$. Setting $2^{1+j_{E}}=$ $\ell E$, we have

$$
T^{E} b=\sum_{s \geq 0} \sum_{j=s+1}^{j_{E}} 2^{-\lambda_{p} j} T_{j} \beta_{j-s}+T^{E} \beta_{0}+\sum_{\sigma \geq 1} \sum_{j=0}^{j_{E}} 2^{-\lambda_{p} j} T_{j} \beta_{j+\sigma} .
$$

The inner product in (3.1) is estimated by

$$
\begin{aligned}
\left|\left\langle T^{E} f, h\right\rangle\right| \leq\left|\left\langle T^{E} g, h\right\rangle\right|+\sum_{s \geq 0}\left|\left\langle\sum_{j=s+1}^{j_{E}} 2^{-\lambda(p) j} T_{j} \beta_{j-s}, h\right\rangle\right| \\
\quad+\left|\left\langle T^{E} \beta_{0}, h\right\rangle\right|+\left|\left\langle\sum_{\sigma \geq 1} \sum_{j=0}^{j_{E}} 2^{-\lambda_{p} j} T_{j} \beta_{j+\sigma}, h\right\rangle\right| \\
=: I+I I+I I I+I V .
\end{aligned}
$$

We estimate the four terms above in order.

As $g$ is bounded, the estimate for the first term is trivial.

$$
\begin{aligned}
I & =\left|\left\langle g, T^{E} h\right\rangle\right| \lesssim\langle f\rangle_{E, p}|E|^{1 / 2}\left\|T^{E} h\right\|_{L^{2}\left(\mathbb{R}^{2}\right)} \\
& \lesssim\langle f\rangle_{E, p}\langle h\rangle_{3 E, 2}|E| \lesssim\langle f\rangle_{E, p}\langle h\rangle_{3 E, q}|E| .
\end{aligned}
$$

This is matches the first term on the right in (3.2).

For term $I I$, we turn to the argument of Seeger, and a consequence of Theorerm 2.8. Recall from $[9,(3.2)]$, that for any $1<p<r<4 / 3$,

$$
\left\|\sum_{j=s+1}^{j_{E}} 2^{-j \lambda_{p}} T_{j} \beta_{j-s}\right\|_{r}^{r} \lesssim 2^{-\frac{1}{2}\left(\frac{r}{p}-1\right) s}\langle f\rangle_{E, p}^{r-p}\|b\|_{p}^{p}, \quad s \geq 0 .
$$

Above, we will take $r=q^{\prime}=\frac{q}{q-1}$. By construction, $\|b\|_{p} \lesssim\langle f\rangle_{E, p}|E|^{1 / p}$. Applying Hölder's inequality, we see that

$$
I I \lesssim 2^{-\frac{1}{2}\left(\frac{1}{p}-\frac{1}{r}\right) s}\langle f\rangle_{E, p}\langle h\rangle_{3 E, q}|E| .
$$

For any choice of $r$ the right side above is summable over $s$. This again matches the first term on the right in (3.2).

For term III, dominate

$$
\left|\left\langle T^{E} \beta_{0}, h\right\rangle\right| \leq \sum_{j \geq 0}\left|\left\langle 2^{-j \lambda_{p}} T_{j} \beta_{0}, h\right\rangle\right| .
$$


Recall from $[9,(3.3)]$ that for any $p<r<4 / 3$

$$
\left\|2^{-j \lambda_{p}} T_{j} \beta_{0}\right\|_{r}^{r} \lesssim 2^{-\frac{1}{2}\left(\frac{r}{p}-1\right) j}\langle f\rangle_{E, p}^{r-p}\|b\|_{p}^{p} .
$$

We arrive at the same estimate as in (3.4):

$$
I I I \lesssim 2^{-\frac{1}{2}\left(\frac{1}{p}-\frac{1}{r}\right) s}\langle f\rangle_{E, p}\langle h\rangle_{3 E, q}|E| .
$$

The term $I V$ is treated differently. Recalling (3.3), estimate $N$ by first inserting a spatial localization term $\mathbf{1}_{3 Q}$ for the inner product associated with $b_{Q}$.

$$
\begin{aligned}
I V \leq \sum_{\sigma \geq 1}\left|\left\langle\sum_{j=0}^{j_{E}} \sum_{Q \in \mathcal{B}: \ell Q=2^{j+\sigma}} 2^{-\lambda_{p} j} \mathbf{1}_{(3 Q)^{c}} T_{j} b_{Q}, h\right\rangle\right| \\
\quad+\left|\left\langle\sum_{\sigma \geq 1} \sum_{j=0}^{j_{E}} \sum_{Q \in \mathcal{B}: \ell Q=2^{j+\sigma}} 2^{-j \lambda_{p}} \mathbf{1}_{3 Q} T_{j} b_{Q}, h\right\rangle\right| \\
:=I_{1}+I_{2} .
\end{aligned}
$$

The term $V_{1}$ is an 'error term,' which is dealt with in Lemma 3.6 below. In particular, the conclusion (3.5) of that Lemma implies upon summation over $\sigma \geq 1$ that

$$
V_{1} \lesssim\langle f\rangle_{E, p}\langle h\rangle_{3 E, q}|E| .
$$

This matches the bound in (3.4).

Turning to $V_{2}$, first recognize that the sum can be reorganized to see that

$$
N_{2} \leq \sum_{Q \in \mathcal{B}}\left|\left\langle T^{Q} b_{Q}, \mathbf{1}_{3 Q} h\right\rangle\right| .
$$

That is, it is close to being the last term in (3.2). Second, recall that $b_{Q}=\left[f-\langle f\rangle_{Q}\right] 1_{Q}$. Thus, for any square $Q \in \mathcal{B}$,

$$
\left|\left\langle T^{Q} b_{Q}, \mathbf{1}_{3 Q} h\right\rangle\right| \leq\left|\langle f\rangle_{Q} \cdot\left\langle T^{Q} \mathbf{1}_{Q}, \mathbf{1}_{3 Q} h\right\rangle\right|+\left|\left\langle T^{Q} f \mathbf{1}_{Q}, \mathbf{1}_{3 Q} h\right\rangle\right| .
$$

The second term on the right is exactly as in (3.2). We don't need to argue further about it. For the first term on the right, we just use the $L^{p}$-norm bound, Hölder's inequality, and the stopping condition to see that

$$
\begin{aligned}
\left|\langle f\rangle_{Q} \cdot\left\langle T^{Q} \mathbf{1}_{Q}, \mathbf{1}_{3 Q} h\right\rangle\right| & \lesssim\langle f\rangle_{E, p}\left\|T^{Q} \mathbf{1}_{Q}\right\|_{q^{\prime}}\left\|\mathbf{1}_{Q} h\right\|_{q} \\
& \lesssim\langle f\rangle_{E, p}|Q|^{1 / q^{\prime}}\left\|\mathbf{1}_{3 Q} h\right\|_{q} .
\end{aligned}
$$

The sum over the disjoint squares $Q \in \mathcal{B}$ is clearly seen to be at most

$$
\langle f\rangle_{E, p} \sum_{Q \in \mathcal{B}}|Q|^{1 / q}\left\|\mathbf{1}_{3 Q} h\right\|_{q^{\prime}} \lesssim\langle f\rangle_{E, p}\langle h\rangle_{3 E, q}|E| .
$$

The proof is complete. 
Lemma 3.6. For every integer $\sigma \geq 1$

$$
\left|\left\langle\sum_{j=0}^{j_{E}} \sum_{Q \in \mathcal{B}_{j+\sigma}} 2^{-\lambda_{p} j} 1_{(3 Q)^{c}} T_{j}^{\lambda_{p}} b_{Q}, h\right\rangle\right| \lesssim 2^{-\sigma}\langle f\rangle_{E, p}\langle h\rangle_{3 E, q}|E|
$$

where $1<p<\frac{4}{3}, 4<q<p^{\prime}$ and $E, f: E \rightarrow \mathbb{C}, h: 3 E \rightarrow \mathbb{C}, j_{E},\left\{b_{Q}\right\}_{Q \in \mathcal{B}}$, and $\left\{\mathcal{B}_{t}\right\}_{0 \leq t<\log _{2} \ell E}$ are as in Lemma 3.1.

Proof. Fixing $p \in\left(1, \frac{4}{3}\right)$, let $T_{j}=T_{j}^{\lambda_{p}}$ for all integers $j \geq 1$. We will show that, uniformly in integers $j \geq 0, \sigma \geq 1, k \geq 2$, and squares $Q \in \mathcal{B}$

$$
\left|\left\langle 1_{\Delta^{k} Q} T_{j} b_{Q}, h\right\rangle\right| \lesssim 2^{\lambda_{p} j} 2^{-k} 2^{-\sigma}\langle f\rangle_{Q, p}\langle h\rangle_{2^{k} Q, q}|Q|
$$

where $\Delta^{k} Q=2^{k} Q \cap\left(2^{k-1} Q\right)^{c}$. Summing on $k \geq 2$ and $0 \leq j \leq j_{E}$, we have

$$
\begin{aligned}
\mid\left\langle\sum_{j=0}^{j_{E}} \sum_{Q \in \mathcal{B}_{j+\sigma}}\right. & \left.2^{-\lambda_{p} j} 1_{(3 Q)^{c}} T_{j} b_{Q}, h\right\rangle \mid \\
& \lesssim 2^{-\sigma} \sum_{k \geq 2} \sum_{j \geq 0} \sum_{Q \in \mathcal{B}_{j+\sigma}}\left|\left\langle 2^{-\lambda_{p} j} 1_{\Delta^{k}{ }_{Q}} T_{j} b_{Q}, h\right\rangle\right| \\
& \lesssim 2^{-\sigma} \sum_{Q \in \mathcal{B}}\langle f\rangle_{Q, p} \inf _{x \in Q}\left[M|h|^{q}\right]^{1 / q}|Q| .
\end{aligned}
$$

An easy application of Hölder's inequality and appealing to the boundedness of the maximal function proves the Lemma.

The claim (3.8) follows from interpolating two separate inequalities. First, appealing to the kernel estimate (2.2), for any measurable and bounded $F, H: \mathbb{R}^{2} \rightarrow \mathbb{C}$

$$
\begin{aligned}
\left|\left\langle 1_{\Delta^{k} Q} T_{j}\left(1_{Q} F\right), H\right\rangle\right| & \leq\left\|1_{\Delta^{k} Q} T_{j}\left(1_{Q} F\right)\right\|_{L^{\infty}}\left\|H 1_{\Delta^{k} Q}\right\|_{1} \\
& \lesssim_{N} 2^{-3 j / 2} 2^{-(N+2) k} 2^{-N \sigma}\left\|1_{Q} F\right\|_{1}\left\|H 1_{\Delta^{k} Q}\right\|_{1} \\
& \lesssim 2^{\frac{j}{2}} 2^{-N k} 2^{-N \sigma}\langle F\rangle_{Q, 1}\langle H\rangle_{2^{k} Q, 1}|Q|, \quad k \geq 2, \quad N>1 .
\end{aligned}
$$

The implied constant depends upon $N$, and below we will specify a choice of $N=N_{p, q}$.

We will also appeal to the norm bound (2.7) on $T_{j}$ on $L^{r}$ for any $1<r<4 / 3$. Namely

$$
\left|\left\langle 1_{\Delta^{k} Q} T_{j}\left(1_{Q} F\right), H\right\rangle\right| \lesssim 2^{\lambda_{r} j} 2^{\frac{2 k}{r^{\prime}}}\langle F\rangle_{Q, r}\langle H\rangle_{2^{k} Q, r^{\prime}}|Q|
$$

As $4<q<p^{\prime}$, we may choose $r=q^{\prime}\left[2-\frac{1}{p}-\frac{1}{q}\right]$, interpolate between (3.9) and (3.10), and set $F=b_{Q}$ and $H=h$ to recover

$$
\left|\left\langle 1_{\Delta^{k} Q} T_{j} b_{Q}, h\right\rangle\right| \lesssim_{N} 2^{\lambda_{p} j} 2^{-k} 2^{-\sigma}\left\langle b_{Q}\right\rangle_{Q, p}\langle h\rangle_{2^{k} Q, q}|Q|
$$


provided $N=N_{p, q} \geq \frac{1}{\frac{1}{p}+\frac{1}{q}-1}\left[1+\frac{2\left(2-\frac{1}{p}-\frac{1}{q}\right)}{\left(q^{\prime}\left[2-\frac{1}{p}-\frac{1}{q}\right]\right)^{\prime}}\right]$. As $\left\langle b_{Q}\right\rangle_{Q, p} \lesssim\langle f\rangle_{Q, p}$, (3.8) follows.

Proof of Theorem 1.2. Let $f, h: \mathbb{R}^{2} \rightarrow \mathbb{C}$ be measurable, bounded, and supported on a (translated) dyadic square $E$. Indeed, we take the cube $E$ so large, with the supports of $f$ and $h$ deeply contained inside $E$, so that we have

$$
\left|\left\langle B_{\lambda_{p}} f, h\right\rangle\right| \leq C\langle f\rangle_{E, p}\langle h\rangle_{E, q}|E|+\left|\left\langle T^{\lambda_{p}, E} f, h\right\rangle\right| .
$$

This is possible to do, as follows from the kernel estimates in Lemma 2.1.

We are now in a position to apply the main recursive estimate of Lemma 3.1. From it, we have

$$
\left|\left\langle T^{\lambda_{p}, E} f, h\right\rangle\right| \leq C\langle f\rangle_{E, p}\langle h\rangle_{E, q}|E|+\sum_{Q \in \mathcal{B}}\left|\left\langle T^{\lambda_{p}, Q}\left(f 1_{Q}\right), \mathbf{1}_{3 Q} h\right\rangle\right| .
$$

The first term on the right we take as the first contribution to our 'sparse' form. We then recursively apply this estimate to the individual terms in the sum over $Q \in \mathcal{B}$. A straightforward recursion produces a collection of sparse cubes $\tilde{\mathcal{S}}$ for which

$$
\left|\left\langle B_{\lambda_{p}} f, h\right\rangle\right| \lesssim \sum_{Q \in \tilde{S}}\langle f\rangle_{Q, p}\langle h\rangle_{3 Q, q}|Q| .
$$

Also, each cube $Q$ there is a set $E_{Q} \subset Q$ with $\left|E_{Q}\right| \geq \frac{1}{100}|Q|$, and the sets $\left\{E_{Q}: Q \in \tilde{\mathcal{S}}\right.$ are pairwise disjoint. But this last bound does not quite meet the definition of a sparse bound. But we can replace the term $\langle f\rangle_{Q, p}$ by $\langle f\rangle_{3 Q, p}$. We then have the sparse bound.

\section{REFERENCES}

[1] Cristina Benea, Frédéric Bernicot, and Teresa Luque, Sparse bilinear forms for bochner riesz multipliers and applications, Transactions of the London Mathematical Society 4 (2017), no. 1, $110-128$.

[2] Lennart Carleson and Per Sjölin, Oscillatory integrals and a multiplier problem for the disc, Studia Math. 44 (1972), 287-299. (errata insert). Collection of articles honoring the completion by Antoni Zygmund of 50 years of scientific activity, III. MR0361607

[3] Michael Christ, On almost everywhere convergence of Bochner-Riesz means in higher dimensions, Proc. Amer. Math. Soc. 95 (1985), no. 1, 16-20. MR796439

[4] _ Weak type endpoint bounds for Bochner-Riesz multipliers, Rev. Mat. Iberoamericana 3 (1987), no. 1, 25-31. MR1008443

[5] A. Córdoba, A note on Bochner-Riesz operators, Duke Math. J. 46 (1979), no. 3, 505-511. MR544242

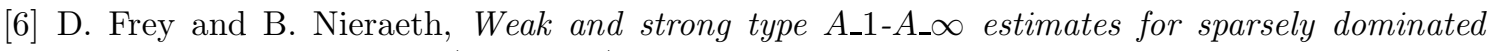
operators, ArXiv e-prints (July 2017), available at 1707.05212.

[7] Lars Hörmander, Oscillatory integrals and multipliers on $F L^{p}$, Ark. Mat. 11 (1973), 1-11. MR0340924

[8] M. T. Lacey, D. Mena Arias, and M. C. Reguera, Sparse Bounds for Bochner-Riesz Multipliers, ArXiv e-prints (May 2017), available at 1705.09375. 
[9] Andreas Seeger, Endpoint inequalities for Bochner-Riesz multipliers in the plane, Pacific J. Math. 174 (1996), no. 2, 543-553. MR1405600

[10] Elias M. Stein and Guido Weiss, Introduction to Fourier analysis on Euclidean spaces, Princeton University Press, Princeton, N.J., 1971. Princeton Mathematical Series, No. 32 . MR0304972

[11] Ana M. Vargas, Weighted weak type $(1,1)$ bounds for rough operators, J. London Math. Soc. (2) 54 (1996), no. 2, 297-310. MR1405057

School of Mathematics, Georgia Institute of Technology, Atlanta GA 30332, USA

E-mail address: rkesler6@math.gatech.edu

E-mail address: lacey@math.gatech.edu 